\title{
Grupos estratégicos e desempenho no setor confeccionista brasileiro
}

\section{Strategic groups and performance in the brazilian clothing sector}

\author{
Lucas Maia dos Santos ${ }^{1}$ \\ Marco Aurélio Marques Ferreira ${ }^{2}$ \\ Bruno Tavares ${ }^{2}$ \\ Daniel Resende Dutra ${ }^{3}$
}

\begin{abstract}
Resumo: Os determinantes do desempenho das empresas têm sido, por muito tempo, interesse central para pesquisadores em gestão estratégica. Nesse sentido, este trabalho foi conduzido com o objetivo de identificar e analisar o desempenho de grupos estratégicos de confecções brasileiras, descrevendo as suas características e comparando-as por indicadores definidos ao longo do estudo. Para isto, foram analisados os dados do ano de 2006 de 510 empresas do setor: calcularam-se os escores de eficiência, utilizando-se a metodologia de análise envoltória de dados; utilizou-se a análise de cluster para identificar agrupamentos e utilizou-se da análise discriminante para validá-los. Constatou-se elevado grau de ineficiência das empresas e o lapso médio de eficiência permite $98,17 \%$ de aumento de produtos, mantendo o mesmo nível de insumos. Foi possível identificar três grupos estratégicos significativamente validados pela análise discriminante, sendo aquele composto basicamente por grandes empresas o que apresentou melhor desempenho, e aquele composto basicamente por micro e pequenas empresas (MPEs) o que apresentou pior desempenho. A presença de agrupamentos estratégicos diferenciados, principalmente por porte, mostra que o setor confeccionista nacional é dividido por barreiras internas inerentes às peculiaridades da maior disponibilidade de recursos em grandes empresas do que nas MPEs. Por isso, esses resultados vão ao encontro da literatura, que aconselha as MPEs a focarem em outras estratégias para não competir com a capacidade de economia de escala das grandes. Do ponto de vista dos grupos estratégicos, isso possibilitará a construção de barreiras de mobilidade interna aos grupos, fortalecendo a competitividade do setor nacional.
\end{abstract}

Palavras-chave: Setor de confecções. Análise de eficiência. Grupos estratégicos. Desempenho.

\begin{abstract}
Determinants of enterprise performance have long been of core interest to strategic management researchers. Therefore, this study was carried out to identify and analyze the performance of strategic groups in the Brazilian clothing industry describing their characteristics to compare with defined variables in the study. Data of 510 enterprises from 2006 were used. The efficiency scores were calculated using Data Envelopment Analysis, cluster analysis was used to identify groups, and Discriminant Analysis was used to validate the clusters. The results showed high degree of inefficiency, and the average deficiency of efficiency results showed the possibility to increasing of $98.17 \%$ in outputs keeping the same level of inputs. It was possible to identify three strategic groups significantly validated by the discriminant analysis, and the group composed basically of large enterprises showed better performance than the one composed basically by micro and small enterprises. The presence of different strategic groups, especially with regards to size, shows that the domestic industry has internal barriers inherent in the peculiarities of the higher resource availability of large companies than that of small companies. Therefore, these results agree with those reported in the literature concerning the suggestion that micro and small businesses should focus on other strategies to avoid competition with the economies of scale of large enterprises. In terms of strategic groups, this allows the building of internal barriers of mobility strengthening the competitiveness of the national industry.
\end{abstract}

Keywords: Clothing sector. Efficiency analysis. Strategic groups. Performance.

\footnotetext{
${ }^{1}$ Instituto Federal de Minas Gerais, Universidade Federal de Minas Gerais - UFMG, Av. Serra da Piedade, 299, Conjunto Morada da Serra, CEP 34515640, Sabará, MG, e-mail: lucas.maia@ifmg.edu.br

2 Departamento de Administração, Universidade Federal de Viçosa - UFV, Av. PH Rolfs, s/n, Centro, CEP 36570-000, Viçosa, MG, e-mail: marcoaurelio@ufv.br; btavares@ufv.br

${ }^{3}$ Instituto Brasileiro de Geografia e Estatística - IBGE, Rua Catanduvas, 341, Renascença, CEP 31130-600, Belo Horizonte, MG, e-mail: danielrdutra@gmail.com
}

Recebido em 18/3/2010 — Aceito em 10/6/2010

Suporte financeiro: Nenhum. 


\section{Introdução}

Os determinantes do desempenho das empresas têm sido, por muito tempo, interesse central para pesquisadores em gestão estratégica. De acordo com Short et al. (2007), pesquisas focadas na explicação do desempenho têm enfatizado determinantes em três níveis de análise: a firma, os grupos estratégicos e a indústria. Todavia, o tema ainda está em construção (MATITZ; BULGACOV, 2011).

Este estudo investigou o desempenho de empresas do setor de confecções brasileiro sobre a abordagem dos grupos estratégicos, a exemplo dos estudos de Lee, Lee e Sangkyu (2002) e Leask e Parker (2004). Alguns pesquisadores em grupos estratégicos discutem que firmas reúnem-se em torno de uma quantidade limitada de similaridades competitivas e algumas dessas oferecem melhor desempenho que outras (McGEE, 2003; McNAMARA; DEEPHOUSE; LUCED, 2003).

O objetivo central da maioria das pesquisas em agrupamento estratégico é averiguar se diferenças significantes entre comportamentos homogêneos de firmas admitem relações com o desempenho delas na indústria (SHORT et al., 2007). O desempenho das organizações pode ser medido de diversas maneiras e alguns trabalhos apresentam diferentes constructos para representá-los, a exemplo dos indicadores de reputação de mercado (FERGUSON; DEEPHOUSE; FERGUSON, 2000); de participação de mercado (PLESHKO; NICKERSON, 2008); de porte (PORTER, 1990); de indicadores econômicos e financeiros (SHORT et al., 2007); e de índices de eficiência técnica de escala (FERREIRA; BRAGA, 2007a, b; FERREIRA; ABRANTES; PEREZ, 2008). Neste trabalho, o desempenho foi quantificado em três dimensões complementares: (I) escores de eficiência; (II) indicadores econômicos; e, por último, (III) porte.

O setor confeccionista, foco de análise deste estudo, tem passado por diversas reestruturações na forma de comércio mundial, desde o término do acordo Multifibras e do Acordo de Têxteis e Confecções da Organização Mundial do Comércio (OMC) no ano 2005. Este evento, associado à utilização intensiva de mão de obra e capacidade de abrigar micro e pequenas empresas (MPEs) instiga estudos para compreender as características e condicionantes do desempenho deste setor.

Sendo um setor constituído, principalmente, por MPEs, a busca de alternativas estratégicas torna-se mais complexa dada as especificidades destas (MONIZ; PAULOS, 2008). Neste segmento, os funcionários executam muitas tarefas no decorrer de todo o processo produtivo, sendo menos especializados. A maior escassez de recursos também restringe a contratação de trabalhadores mais qualificados. As economias da divisão de tarefas também se aplicam à gestão empresarial, sendo que o gestor de MPEs envolve-se em tarefas operacionais e cotidianas, reduzindo o tempo para funções de planejamento e análise ambiental (LEONE, 1999; TAVARES; FERREIRA; LIMA, 2008).

Dessa forma, este trabalho foi conduzido com o objetivo de identificar e analisar o desempenho de grupos estratégicos de confecções brasileiras, caracterizando e comparando este desempenho por indicadores que serão posteriormente descritos. Mais especificamente, buscou-se a) mensurar o grau de eficiência técnica e de escala por meio da relação entre as variáveis financeiras e desempenho nessas confecções; e b) identificar, em razão de homogeneidades e disparidades estruturais e estratégicas, diferentes grupos de organizações na indústria nacional de confecções.

\section{Revisão teórica}

\subsection{Caracterização do setor de confecções}

Vários autores, a exemplo de Saraiva, Pimenta e Corrêa (2001), Nordas (2004) e Doig (2005), descrevem que o setor confeccionista passou por um rápido processo de reestruturação, visando manter seu potencial competitivo no mercado nacional.

Notadamente, desde o final da década de 1990, existe a tendência de substituição da produção nacional por produtos importados. Adicionalmente, a abolição das cotas de importação e exportação desses produtos pela OMC, em 2005, mudou a ordem do comércio mundial. Assim, produtos da cadeia têxtil receberam a mesma aplicabilidade de outros produtos, possibilitando maior competitividade para países com baixo custo de produção como China, Tailândia, Hong Kong e Índia (BRODNER; LATNIAK, 2002; ADHIKARI; YAMAMOTO, 2007; ASLESEN, 2008).

Nesse cenário, muitas empresas promoveram ajustes no sentido de tornarem-se mais competitivas. Outras, porém, têm experimentado redução comparativa do desempenho, justificada em parte por não agregarem valor suficiente ou mesmo em decorrência do déficit tecnológico (AGIS; GOUVEIA; VAZ, 2002; DOIG, 2005).

Segundo Unicamp (UNIVERDADE..., 2008) e Abravest (ASSOCIAÇÃO..., 2009), o setor de confecções brasileiro é intensivo em capital humano, exige pouca infraestrutura para instalação das fábricas e baixa qualificação da força produtiva. Portanto, é marcado pela migração da produção para locais em que o custo da mão de obra é relativamente menor.

A característica estrutural básica da indústria de confecção, em nível mundial, é a grande heterogeneidade das unidades produtivas em termos de tamanho, escala de produção e padrão tecnológico (PELISSARI; GONZALEZ; VANALLE, 2011). 
Segundo Brodner e Latniak (2002), Nordas (2004) e Pelissari, Gonzalez e Vanalle (2011), a indústria de confecção tem se caracterizado pela fragilidade das barreiras à entrada, tanto em relação à tecnologia como ao valor dos investimentos. A técnica de produção é amplamente conhecida e o equipamento utilizado - a máquina de costura - é de operação simples e custo reduzido, o que facilita a entrada de produtores de menor porte na indústria. O elevado número de MPEs no setor justifica-se, ademais, pelo fato de que, ao contrário de outros segmentos industriais, o baixo custo da mão de obra ainda exerce grande influência na decisão de investir e nas estratégias de localização dos empreendimentos.

\subsection{Fatores condicionantes da eficiência no setor de confecções}

A compreensão do desempenho organizacional e das vantagens competitivas que distinguem o sucesso das organizações tem sido o centro das discussões de diversos trabalhos acadêmicos ao longo da última década e, portanto, tem instigado o esforço investigativo de diversos pesquisadores na busca da compreensão dos seus elementos orientadores (BURDEN; PROCTOR, 2000; MA, 2000; FAHY, 2000; BARNEY, 2001, LIN, 2003; FAHY; FARRELLY; QUESTER, 2004; DENRELL, 2004, COUSINS, 2005; PORTER; KRAMER, 2006; LIAO; HU, 2007; FERREIRA; BRAGA, 2007a). O desempenho pelo uso de fatores materiais, humanos e criativos tem estado presente na maior parte das descobertas.

Segundos dados de Moniz e Paulos (2008), Abit (ASSOCIAÇÃO..., 2009) e Abravest (ASSOCIAÇÃO..., 2009), nas fases anteriores à costura, como não há necessidade de manusear o tecido, houve notórios avanços tecnológicos cujos benefícios se traduzem na redução do tempo no processo de produção e no desperdício de tecidos, além de flexibilidade para a alteração de modelos. Porém esses procedimentos ainda são pouco encontrados na maioria das confecções, formadas basicamente por MPEs (WOLL et al., 2007; MONIZ; PAULOS, 2008; SEBRAE, 2009).

Não obstante, tais avanços, principalmente, nas fases desenho e corte, os entraves à automação da fase da costura têm exigido, por parte dos países industrializados, a adoção de estratégias competitivas baseadas em outros fatores além da modernização industrial (CORRÊA; PIMENTA, 2006; ASLESEN, 2008; MONIZ; PAULOS, 2008). A permanência do "gargalo tecnológico" nas fases de costura e montagem das indústrias de confecções tem impulsionado estratégias empresariais baseadas na subcontratação, enquanto estratégia que viabiliza a flexibilidade e a rapidez da produção por meio da montagem de uma rede de unidades produtoras coordenadas por uma empresa central.

Por outro lado, a terceirização vem sendo confundida com informalização da mão de obra, sendo que muitas empresas, especialmente as de menor porte, tendem a operar onde haja mão de obra abundante, de menor custo, incentivos fiscais e menor carga tributária. Isto reflete a busca da redução de custos e ganhos de competitividade via absorção das vantagens propiciadas pelos salários mais baixos vigentes naqueles países. Este caminho, nomeado por Woll et al. (2007) como de "estrada baixa", encobre problemas sociais de longo prazo provocados por baixos salários e baixa taxas de eficiência, afetando, principalmente, as MPEs que possuem importante participação na economia nacional.

A grande heterogeneidade neste setor mostra que a estratégia, entre MPEs e grandes empresas, configura-se como concorrência, embora as empresas menores devam ajustar-se a nichos nos quais não sofram concorrência direta das grandes. $\mathrm{O}$ posicionamento das MPEs num mercado mais restrito, de modo a construir a fidedignidade dos clientes, pode ser uma alternativa estratégica (MA, 2000; PORTER; KRAMER, 2006). Como discutido por Agis, Gouveia e Vaz (2002), uma alternativa refere-se à posição estratégica no mercado globalizado e competitivo, forçando o setor a buscar, ainda, enfoques cooperativos para as microempresas.

Outro ponto a se discutir é que a adoção de estratégias que eliminam a necessidade de economia de escala pode potencializar o desenvolvimento da pequena empresa. Por exemplo, pode-se atuar em mercados cujos produtos são de difícil padronização; atuar em mercados limitados a pequenos nichos que possuem demanda restrita ou fornecer produtos cujas matérias-primas são dispersas ou de difícil transporte (PORTER; KRAMER, 2006; LIAO; HU, 2007).

\subsection{Abordagem dos agrupamentos estratégicos}

De acordo com Joyce (2003) e Short et al. (2007), agrupamentos ou grupos estratégicos podem ser definidos como um conjunto de empresas que são mais homogêneas em ações dentro do mesmo ambiente industrial.

Nessa ótica, grupos estratégicos podem ser identificados e classificados em todos os setores em que um número significativo de organizações participa. Neste estudo, inicialmente, foram obtidos os escores de eficiência do setor analisado e, baseado nestes, foram obtidos três grupos distintos por análise de cluster. A maioria dos pesquisadores forma grupos estratégicos por intermédio da técnica multivariada de análise de cluster a partir de variáveis que correspondam a componentes estratégicos 
(FERGUSON; DEEPHOUSE; FERGUSON, 2000; JOHNSON et al., 2011).

Nas últimas duas décadas, pesquisas em grupos estratégicos estão centradas sobre três principais pontos: emergência de grupos estratégicos, diferença entre desempenho dos grupos e estabilidade da estrutura dos grupos (LEE; LEE; SANGKYU, 2002, p. 728). Este estudo focou o segundo ponto, buscando identificar diferenças de desempenho entre os agrupamentos obtidos.

Segundo Ferguson, Deephouse e Ferguson (2000), Joyce (2003), Killstrom (2005) e Short et al. (2007), existe a lógica suportando a expectativa de que grupos estratégicos variam em desempenho. O processo de agrupamentos estratégicos provê a oportunidade de testar se a diferença em desempenho observada entre as empresas pode ser explicada, ao menos, pelas associações ao grupo estratégico e, dessa forma, pelo tipo de estratégia adotada. Diversos estudos citam a ligação entre desempenho e agrupamento estratégico entre eles Lee, Lee e Sangkyu (2002), Shergill (2002), McGee (2003), McNamara, Deephouse e Luced (2003), Leask e Parker (2004) e Killstrom (2005),

Ferreira, Abrantes e Perez (2008), Short et al. (2007), Ferreira e Braga (2007a) e Pleshko e Nickerson (2008). Em particular, na literatura industrial, as firmas devem ser protegidas por barreiras de mobilidade interna, de entrada e saída. Essas barreiras impedem movimentos dentro da mesma indústria ou de entrada de firmas externas, em razão da necessidade de investimento, diferenças de porte, lucratividade, rentabilidade ou de disponibilidade de recursos. Essas barreiras resultam de ações coletivas que protegem cada grupo estratégico de competição externa (JOYCE, 2003; McGEE, 2003; SHORT et al., 2007; PLESHKO; NICKERSON, 2008).

Barreiras de mobilidade interna são classificadas na literatura como aquelas relacionadas ao mercado, como propaganda, força de vendas, dimensão e aquelas relacionadas aos ativos (McGEE, 2003).

Killstrom (2005, p. 11), em seu estudo relatou significante diferença entre grupos estratégicos em termos de rentabilidade, eficiência e retorno sobre o investimento. Dependendo das barreiras de entrada, saída e mobilidade em uma mesma indústria, as oportunidades não são igualmente distribuídas e alguns segmentos podem ser mais eficientes que outros.

Os benefícios de uma análise condicionada pela composição de grupos estratégicos estão ligados à exploração das características físicas e comportamentais das organizações que compõem um mesmo escopo estratégico, visando interpretar a razão de suas diferenças em dado atributo. Esse atributo pode ser desempenho financeiro, crescimento operacional, parcela de mercado ou eficiência. No presente estudo, buscar-se-á a diferenciação pelo desempenho financeiro e pela eficiência, a exemplo de Lee, Lee e Sangkyu (2002) e Killstrom (2005).

\section{Metodologia}

Este estudo baseou-se em dados secundários disponibilizados pelo IBGE (INSTITUTO..., 2009), referentes a 510 empresas brasileiras do setor de confecções no ano de 2006, sendo estes os dados mais recentes do setor.

Para consecução dos objetivos, quatro métodos foram utilizados para o desenvolvimento deste estudo: primeiro, fez-se uso da metodologia de Análise Envoltória de Dados (CHARNES; COOPER; RHODES, 1978; BANKER, 1984; BANKER; CHARNES; COOPER, 1984) para calcular os escores de eficiência; em seguida, utilizou-se a análise de cluster (MAROCO, 2003; HAIR, 2005) para identificar agrupamentos dentro do setor; adicionalmente, foi acrescentado um procedimento de análise discriminante com a intenção de validar os clusters obtidos (MAROCO, 2003); ademais, foi utilizado no decorrer das análises, o teste do Quiquadrado para comparação entre variáveis nominais e o teste ANOVA para comparação de médias, sobre a hipótese de amostras aleatórias não independentes. Esses testes foram necessários para algumas comparações entre subamostras, como a validação das diferenças entre as variáveis utilizadas para interpretar os clusters pela ANOVA e diferenciação entre o porte das empresas utilizando-se o teste Quiquadrado. Os procedimentos de ambos os testes podem ser resgatados em Pestana e Gageiro (2000), Maroco (2003) e Hair (2005).

\subsection{Mensuração da eficiência}

No presente trabalho, a mensuração da eficiência foi realizada com a utilização da metodologia de Análise Envoltória de dados (DEA - sigla do inglês Data Envelopment Analysis), com orientação para aumento proporcional dos produtos. Esta orientação foi aplicada neste estudo tomando por base que as empresas de confecções devem visar o aumento dos seus produtos do modelo analítico, mantendo fixa a quantidade de insumos utilizados.

Adaptado de Charnes, Cooper e Rhodes (1978), o modelo orientado à expansão dos produtos, considerando retornos constantes de escala (RCE), foi definido pelo seguinte problema de programação linear (Equação 1): 


\section{$\operatorname{Max} \theta^{R C E}$}

Sujeito a:

$$
\begin{aligned}
& \sum_{j=1}^{n} \lambda_{j} x_{i j} \leq x_{i 0,}, i=1,2, \ldots, m \\
& \sum_{j=1}^{n} \lambda_{j} y_{r j} \geq \theta^{R C E} y_{i 0,}, r=1,2, \ldots, S \\
& \sum_{j=1}^{n} \lambda_{j} \geq 0, \forall j
\end{aligned}
$$

Considerando que as DMUs (unidades tomadoras de decisão - do inglês decision making units - que neste estudo são as confecções) utilizem $m$ insumos e $s$ produtos, $j$ está associado à $\mathrm{DMU}_{0}$ analisada e $n$ corresponde ao número total de DMUs; $x_{i j}$ é a quantidade consumida do insumo $i(i=1,2 \ldots, m)$ pela $\mathrm{DMU}_{j} ; y_{r j}$ é a quantidade produzida do produto $r(r=1,2,3 \ldots s)$ pela $\mathrm{DMU}_{\mathrm{j}} ; \lambda_{j}$ é o peso da $\mathrm{DMU}_{j}$ na composição da $\mathrm{DMU}_{0}$ virtual analisada (projeção na fronteira eficiente).

O problema apresentado na Equação 1 é resolvido $n$ vezes, sendo uma vez para cada DMU e, como resultado, apresenta os valores de $\theta^{R C E}$ e $\lambda_{j}$, sendo $\theta^{R C E}$ o escore de eficiência da DMU sob análise e $\lambda_{j}$ fornece as DMUs eficientes que servem de referência ou benchmark para a DMU $j$ analisada. Neste estudo, os escores de eficiência serão mostrados, percentualmente, em uma escala de $0 \%$ a $100 \%$.

No intuito de incorporar a possibilidade de retornos variáveis à escala, Banker, Charnes e Cooper (1984) propuseram o modelo de retornos variáveis à escala (RVE) da análise envoltória de dados, introduzindo a restrição de convexidade $\sum_{j=1}^{n} \lambda_{j}=1$ no modelo RCE, apresentado no Problema de Programação Linear da Equação 4. O modelo com retornos variáveis à escala de Banker, Charnes e Cooper (1984), com orientação ao aumento dos produtos pode ser definido por (Equação 2):

\section{$\operatorname{Max} \theta^{R V E}$}

\section{Sujeito a:}

$$
\begin{aligned}
& \sum_{j=1}^{n} \lambda_{j} x_{i j} \leq x_{i 0,}, \quad i=1,2, \ldots, m \\
& \sum_{j=1}^{n} \lambda_{j} y_{r j} \geq \theta^{R V E} y_{i 0,} r=1,2, \ldots, s \\
& \sum_{j=1}^{n} \lambda_{j}=1 \\
& \sum_{j=1}^{n} \lambda_{j} \geq 0, \forall j
\end{aligned}
$$

Novamente, considerando que as DMUs utilizem $m$ insumos e $s$ produtos, $j$ está associado à $\mathrm{DMU}_{0}$ analisada e $n$ corresponde ao número total de DMUs; $x_{i j}$ é a quantidade consumida do insumo $i(i=1,2 \ldots, m)$ pela $\mathrm{DMU}_{i} ; y_{r j}$ é a quantidade produzida do produto $r(r=1,2,3 . . s)$ pela $\mathrm{DMU}_{j} ; \lambda_{j}$ é o peso da $\mathrm{DMU}_{j}$ na composição da $\mathrm{DMU}_{0}$ virtual analisada (projeção na fronteira eficiente).

O modelo RVE, apresentado no problema de programação linear da Equação 2, é menos restritivo do que o modelo RCE e permite, de acordo com Banker e Thrall (1992), decompor a eficiência técnica em eficiência de escala e "pura" eficiência técnica. Banker, Charnes e Cooper (1984) citam que o modelo RVE leva em consideração a possibilidade de que a produtividade média do tamanho de escala mais produtivo possa não ser alcançado por outros tamanhos de escala em que uma determinada DMU possa estar operando.

Em relação à eficiência de escala, torna-se necessário estimar a eficiência das DMUs, utilizando-se tanto o modelo RCE como o RVE. A ineficiência de escala é evidenciada quando existem diferenças nos escores desses dois modelos, o que determina um retorno variável, que exige para sua correta classificação, entre não crescente e não decrescente. Embora se tenha optado por essa abordagem, vale ressaltar a existência de diversas alternativas de mensuração da eficiência de escala, discorridas em Banker, Charnes e Cooper (1984) e Banker e Thrall (1992).

A eficiência de escala foi calculada pela relação entre eficiência técnica sobre o pressuposto de retornos constantes à escala (RCE) e eficiência técnica pura sobre o pressuposto de retornos variáveis à escala (RVE), conforme a Equação 3, adaptada de Banker e Thrall (1992).

$$
E S=\frac{\theta^{R C E}}{\theta^{R V E}}
$$

Na composição da matriz de insumos e produtos, buscou-se utilizar quesitos já validados pelos estudos de Ramcharran (2001) e Ferreira e Braga (2007a, b). Na compreensão dos elementos que estão relacionados à eficiência no setor de confecções, especificamente, é importante compreender, na perspectiva de Fahy (2000), alguns fatores centrais caracterizadores, dos quais merecem destaque:

- os recursos que estão relacionados à sustentabilidade do negócio e a geração de vantagens competitivas;

- o sistema de produção ou controle desses recursos pela organização; e

- as escolhas estratégicas da organização na identificação e usos dos recursos-chave para a maximização dos retornos.

Nessa direção, o modelo teórico desse trabalho se baseia na perspectiva de que a firma se orienta para o alcance de resultados desejados, frutos de esforços 
gerenciais, visando enquanto medida comum o retorno financeiro, vez que os diversos outros objetivos podem variar de acordo com as perspectivas pessoais de cada empresário.

A escolha dos produtos no modelo de eficiência é por certo o ponto central do modelo analítico vez que os resultados são a base do modelo de desempenho e devem permitir um alinhamento homogêneo nas diversas unidades analisadas que compõem a amostra. Nesse ponto, torna-se relevante destacar a necessidade de escolha de um produto que atenda a algumas especificações mínimas, tais como: a) confiabilidade; b) unidade comum de mensuração; e c) capacidade de discriminar o desempenho em processo de eficiência relativa.

$\mathrm{O}$ uso de registrados quantitativos legais, a exemplo de relatórios financeiros, que são em última instância os dados oficiais da organização, atende ao primeiro quesito. Os dois outros quesitos poderiam ser atendidos naturalmente, por unidade física de produção, entretanto, no caso do setor de confecção a agravante está no fato de as empresas produzirem uma cesta de diferentes produtos, entre uma infinidade de alternativas, e não apenas um produto, o que impossibilitaria a utilização de variáveis físicas. Portanto, se faz necessário o uso de variável que atenda à exigência de conversão da produção a uma unidade de ponderação comum, visando a homogeneidade entre as DMUs, mesmo com todos os custos advindos dessa escolha. Nessa ótica, a exemplo de outros trabalhos como os de Fath (2000), Ramcharran (2001) e Ferreira e Braga (2007), o faturamento (receita) cumpre bem esse papel.

Desse modo, foi escolhido para mensuração da eficiência a receita total como variável para produto e as variáveis gasto total com pessoal, consumo de matéria-prima e ativo total como variáveis para insumos.

A receita total indica um produto de todos os esforços utilizados pela empresa. Gasto com pessoal é uma variável importante, pois, como afirmou Brodner e Latniak (2002), Adhikari e Yamamoto (2007) e Aslesen (2008), o setor confeccionista é intensivo em mão de obra. A utilização da matéria-prima como variável para insumo também é importante, pois, o tecido é a principal matéria-prima e o modo como cada empresa o utiliza pode determinar sua eficiência, do ponto de vista de redução dos desperdícios. Por último, o ativo total foi utilizado como uma proxy de tamanho.

Neste estudo foram expostos os coeficientes de eficiência para ambos RCE e RVE, pois não foi encontrado na literatura algo que justifique a predominância de apenas um dos retornos de escala (constante ou variável). Alguns dos testes para prevalência de retornos de escala são encontrados em Banker, Charnes e Cooper (1984) e Banker e Thrall (1992).

\subsection{Análise de cluster}

De acordo com Hair (2005), a análise de cluster é o nome para o grupo de técnicas multivariadas que tem o objetivo de agrupar objetos baseando-se nas características que eles possuem. Assim, os objetos classificados em um mesmo grupo são muito similares aos outros com relação a alguns critérios predeterminados. Os clusters resultantes devem exibir alta homogeneidade interna e alta heterogeneidade externa. Assim, se a classificação foi satisfatória, os objetos nos clusters serão próximos quando plotados geometricamente. A partir dos escores de eficiência, foi realizada a análise de cluster, formando grupos ou conglomerados semelhantes. Por ser este o mesmo propósito perseguido pela análise de agrupamentos estratégicos, torna-se, portanto, o procedimento multivariado mais adequado aos objetivos do trabalho.

Este estudo utilizou o método não hierárquico k-means, citado em Maroco (2003) e Hair (2005), como a técnica para particionar os grupos de confecções. Os métodos não hierárquicos inserem objetos nos clusters, uma vez que o número de clusters já foi especificado.

Segundo Maroco (2003) e Hair (2005), nesse procedimento é realizada a partição inicial dos sujeitos em $K$ clusters definidos pelo pesquisador e, em seguida, procede-se ao cálculo dos centroides para cada um dos K clusters e o cálculo da distância euclidiana dos centroides a cada sujeito na base de dados. O emprego do quadrado da distância euclidiana, como medida de semelhança entre as observações, é expresso matematicamente por (Equação 4):

$$
D_{i, j}=\left[\sum_{k=1}^{p}\left(x_{i k}-x_{j k}\right)^{2}\right]^{\frac{1}{2}}
$$

em que, para $p$ variáveis, a distância euclidiana $D_{i, j}$ é calculada entre sujeitos $i$ e $j$ e o valor $x_{i k}$ é o valor da variável $k$ no sujeito $i$ e $x_{j k}$ é o valor da variável $k$ para o sujeito $j$.

Se a distância euclidiana for próxima a zero, significa que os objetos comparados são similares. Por fim, agrupam-se os sujeitos aos clusters cujos centroides se encontram mais próximos, conforme a distância euclidiana (MAROCO, 2003).

De acordo com Maroco (2003), esse método apresenta como principal vantagem, relativamente aos métodos hierárquicos, a facilidade com que são aplicados a matrizes de dados muito grandes, uma vez que não é preciso calcular e armazenar uma nova matriz de dessemelhança em cada passo do algoritmo.

Adicionalmente, os métodos não hierárquicos são capazes de reagrupar os sujeitos num cluster diferente 
daquele em que estes foram inicialmente incluídos, o que não acontece com os métodos hierárquicos nos quais a inclusão de um sujeito num determinado cluster é definitiva. A probabilidade de classificação errada de um determinado sujeito num determinado cluster é, pois, menor nos métodos não hierárquicos (MAROCO, 2003).

É comum nas análises em ciências sociais aplicadas, a exemplo da administração e economia, dar nomes a cada agrupamento de acordo com suas características. É comum realizar também uma análise descritiva comparativa, para a validação dos agrupamentos, tomando como referência as variáveis selecionadas.

\subsection{Análise discriminante}

A análise discriminante é uma técnica de estatística multivariada que permite conhecer as variáveis mais importantes para a discriminação de grupos, classificar novos casos de acordo com a segmentação já conhecida, identificar grupos similares, validar a análise de cluster e confirmar os resultados da análise de componentes principais (PESTANA; GAGEIRO, 2000; MAROCO, 2003; HAIR, 2005).

A seleção das $m$ variáveis discriminantes de um conjunto de $p$ variáveis $(p>m)$ é obtida por recurso à estatística $\Lambda$ de Wilks para cada uma das $p$-variáveis (Equação 5):

$$
\Lambda_{p}=\frac{S Q E_{p}}{S Q T_{p}}
$$

em que $S Q E$ é a soma de quadrados dos erros (dentro dos grupos) e $S Q T$ é a soma dos quadrados totais. A distribuição exata de $\Lambda$ não é conhecida, mas a transformação (Equação 6):

$$
F=\left(\frac{1-\Lambda}{\Lambda}\right) \times\left(\frac{N-p-1}{p}\right)
$$

que pode ser usada no caso de dois grupos, possui distribuição F-Snedecor, com $p$ e $N-p-1$ graus de liberdade.

As hipóteses sob teste são $H_{0}: \mu_{1}=\mu_{2}=\ldots=\mu_{k i}$ contra $\mathrm{H}_{0}$ existe pelo menos uma média diferente das restantes para a variável $i(i=1, \ldots, p)$. Se rejeitar $\mathrm{H}_{0}$ para a variável $i$, pode-se concluir que essa variável discrimina entre os grupos. Contudo, o uso de $p$ estatísticas $\mathrm{F}$ tem um erro de tipo I acrescido de $p \times \alpha \mathrm{e}$, portanto, torna-se necessário comparar o p-valor com $\alpha / p$.

Para desenvolver este procedimento, a amostra foi dividida em duas subamostras, sendo uma utilizada para desenvolver a função discriminante e a outra para testar a função. Esse procedimento é uma maneira de se testar a função obtida, denominada cross-validation. As subamostras foram obtidas aleatoriamente por meio de software estatístico (MAROCO, 2003).

\section{Resultados}

\subsection{Eficiência técnica do setor confeccionista}

Inicialmente, foram calculados os escores de eficiência das 510 empresas. Na Tabela 1, estão apresentadas as variáveis empregadas no modelo de eficiência, juntamente com suas estatísticas descritivas.

Como a amostra é bastante diversificada e foram consideradas empresas de diferentes portes, constata-se a partir da Tabela 1 que as variáveis utilizadas no modelo de eficiência possuem grande variabilidade e amplitude, confirmadas pelos valores de mínimo e máximo, bem como pelo desvio padrão. Destaca-se, portanto, a ampla abrangência da amostra, que contempla, em um só escopo, micro, pequenas, médias e grandes empresas.

Pela média dos escores de eficiência técnica, expostos na Tabela 2, é possível visualizar o grau de ineficiência das organizações, possibilitando aos agentes públicos e privados traçarem políticas que estimulem a melhoria do desempenho do setor. Observa-se que apenas 5,5\% das unidades foram classificadas como eficientes a retornos constantes e $12,5 \%$ a retornos variáveis. Melhor desempenho foi observado quanto à escala, em que 52\% das empresas foram classificadas como eficientes.

Da mesma maneira, a Tabela 2 mostra que a média para eficiência técnica a retornos constantes foi de $48,40 \%$ e para eficiência técnica a retornos variáveis foi de $54,54 \%$. Para eficiência de escala, a média foi de $90,75 \%$. Os valores médios de eficiência técnica encontram-se distantes dos $100 \%$, evidenciando que existem poucas empresas que se destacam por sua produtividade, comparativamente, das outras empresas

Tabela 1. Estatística descritiva das variáveis empregadas no modelo de eficiência.

\begin{tabular}{lcrrrr}
\hline \multicolumn{1}{c}{ Variáveis (em mil) } & & Média & Mínimo & Máximo & Desvio padrão \\
\hline Receita total & Produto & $9.395,91$ & 79,24 & $660.266,70$ & $38.765,50$ \\
Ativo total & Insumo & $13.109,10$ & 3,32 & $161.983,00$ & $100.197,00$ \\
Gasto total de pessoal & Insumo & $1.981,41$ & 14,36 & $118.934,90$ & $80.11,87$ \\
Consumo de matéria-prima & Insumo & $3.657,87$ & 0,12 & $201.969,70$ & $12.283,70$ \\
\hline
\end{tabular}

Fonte: resultados da pesquisa. 
em análise. Este estudo não investigará quais são os motivos das diferenças na produtividade, mas apenas indicará que existem essas diferenças.

Para apresentar o lapso de eficiência técnica e de escala das confecções, foi utilizado o escore médio de eficiência técnica no modelo com retornos variáveis à escala, tomando-se como referência o indicador utilizado por Ferreira e Braga (2007) (Equação 7):

$$
\left[\left(\frac{1}{\text { escore }}\right)-1\right] \times 100
$$

Desse modo, é possível dizer que o lapso médio de eficiência técnica nas confecções possui capacidade de aumento de outputs na ordem de $98,17 \%$ mantendo o mesmo nível de input. A grande ineficiência técnica pode ser uma das explicações para a baixa competitividade dos produtos brasileiros que, por sua vez, vêm sofrendo com a entrada de confeccionados estrangeiros, padronizados e de baixo custo, de acordo com as considerações de Saraiva, Pimenta e Corrêa (2001). Da mesma maneira a grande ineficiência pode ser associada aos problemas inerentes à gestão das micro e pequenas empresas, bem como, à escassez de recursos, já que este setor, segundo o IBGE (INSTITUTO..., 2009) e Abravest (ASSOCIAÇÃO ..., 2009), tem grande presença de empresas deste porte.

Assim, com relação ao porte, percebe-se, pela Tabela 3, que a amostra é composta por grande número de empresas de micro e pequeno porte (75\%), corroborando as afirmações de Abravest (ASSOCIAÇÃO..., 2009) sobre as características do setor confeccionista nacional. A pequena barreira de entrada por meio da produção de confeccionados padronizados, com mão de obra pouco qualificada, baixo custo e ampla demanda, explica a grande inserção de MPEs neste setor. Essa afirmação vai ao encontro dos achados de Tavares, Ferreira e Lima (2008).

De posse desses resultados, realizou-se o teste ANOVA para comparar as diferenças entre escores de eficiência para diferentes portes e a eficiência a retornos constantes foi o único escore com significância superior a 5\%, não sendo significativamente diferente entre os portes.

Observa-se que as empresas de grande porte são mais eficientes a retornos variáveis, com uma média de eficiência de $86,7 \%$, e as MPEs, menos eficientes com um escore médio de $51,8 \%$. Porém, verifica-se o oposto, quando se compara a eficiência de escala, pois, neste quesito, as MPEs possuem melhor desempenho. Era esperada a baixa eficiência técnica das MPEs de acordo com as considerações de Woll et al. (2007) e Aslesen (2008) sobre a pouca qualificação da mão de obra utilizada no setor confeccionista, ao "gargalo tecnológico" das etapas de corte e costura, além da reconhecida limitação de recursos para investimentos do setor de micro e pequenas empresas brasileiras.

\subsection{Análise dos grupos estratégicos}

No intento de aprofundar o entendimento intraindústria, utilizou-se a análise de cluster para identificar agrupamentos dentro do setor analisado. Como Joyce (2003) e Short et al. (2007) afirmaram que grupos estratégicos variam em desempenho, torna-se instigante identificar essas diferenças.

Para formação dos clusters, foram utilizados os escores de eficiência anteriormente calculados. Por meio do estudo do comportamento das variáveis selecionadas, buscou-se investigar a existência ou não de empresas que apresentam comportamentos

Tabela 2. Estatística descritiva dos escores de eficiência.

\begin{tabular}{lccccc}
\hline $\begin{array}{c}\text { Escores de } \\
\text { eficiência }\end{array}$ & $\begin{array}{c}\text { Unidades eficientes } \\
(\boldsymbol{\%})\end{array}$ & $\begin{array}{c}\text { Média } \\
(\boldsymbol{\%})\end{array}$ & $\begin{array}{c}\text { Desvio padrão } \\
(\boldsymbol{\%})\end{array}$ & $\begin{array}{c}\text { Máximo } \\
(\boldsymbol{\%})\end{array}$ & $\begin{array}{c}\text { Mínimo } \\
(\boldsymbol{\%})\end{array}$ \\
\hline RCE & 5,5 & 48,40 & 19,44 & 100,00 & 8,10 \\
RVE & 12,5 & 54,54 & 22,57 & 100,00 & 8,30 \\
ESCALA & 52,5 & 90,75 & 13,10 & 100,00 & 24,72 \\
\hline
\end{tabular}

Fonte: resultados da pesquisa.

Tabela 3. Eficiência por porte.

\begin{tabular}{cclcccc}
\hline Porte & Amostra (\%) & Eficiência & Média & Mínimo & Máximo & Desvio padrão \\
\hline \multirow{2}{*}{ MPEs } & \multirow{2}{*}{75} & RVE & 51,28 & 10,60 & 100,00 & 21,72 \\
& & ESCALA & 93,91 & 24,72 & 100,00 & 9,31 \\
\hline \multirow{2}{*}{ Médias } & \multirow{2}{*}{21} & RVE & 60,12 & 8,30 & 100,00 & 20,72 \\
& & ESCALA & 85,66 & 26,31 & 100,00 & 14,60 \\
\hline \multirow{2}{*}{ Grandes } & \multirow{2}{*}{4} & RVE & 86,67 & 35,80 & 100,00 & 17,15 \\
& & ESCALA & 57,94 & 33,26 & 83,48 & 13,42 \\
\hline
\end{tabular}

Fonte: resultados da pesquisa. 
idênticos ou assemelhados com relação aos escores de eficiência e, a partir deste enfoque, caracterizar e interpretar os grupos por outras variáveis. Essas outras variáveis foram:

- Rentabilidade do ativo (ROA): Segundo Silva (2006) e Matarazzo (2008), índices de rentabilidade mostram qual a rentabilidade dos capitais investidos e, portanto, o grau de êxito econômico da empresa. É uma medida potencial de geração de lucro da parte da empresa;

- Giro do ativo: É a razão entre vendas líquidas e ativo. Segundo Matarazzo (2008), este índice mede o volume de vendas de uma empresa em relação ao capital total investido, pois não é correto observar o desempenho de uma empresa apenas pelo valor absoluto de suas vendas. No setor de confecções, no qual a redução dos custos é importante para a competitividade internacional, o giro é uma variável fundamental para elevação da rentabilidade quando a dificuldade pelo ganho de margem líquida é maior;

- Margem líquida: proxy de agregação de valor. Formada pela razão entre lucro líquido e receita de vendas. É formado pelo valor das vendas menos o custo do produto vendido. Indica quanto a empresa está agregando de valor; e

- Pessoal ocupado: proxy de tamanho. Segundo Souza (2007) e Sebrae (2009), além do valor do faturamento bruto, o número de pessoal ocupado é o principal critério atualmente utilizado para diferenciação entre porte das empresas.

Desta forma, foi realizada uma análise de variância (ANOVA) buscando identificar diferenças significativas entre os clusters para essas variáveis. Como demonstrado na Tabela 4 , as variáveis mostraram-se significativas a 5\% para diferenciar os grupos formados. Essas variáveis foram utilizadas na formação dos grupos porque não se adequaram ao procedimento de análise de cluster.

Optou-se, neste estudo, por utilizar apenas três clusters, determinando a classificação entre grupo inferior, intermediário e outro superior quanto ao desempenho. As médias dos indicadores entre os grupos estão na Tabela 5. Observa-se que o grupo
1 possui $60 \%$ das empresas eficientes para RVE e nenhuma empresa eficiente para RCE e eficiência de escala. Por outro lado, no grupo 2, 82,6\% das empresas são eficientes em escala, mas nenhuma mostrou ser eficiente para os escores de RCE e RVE.

A Tabela 6 apresenta a análise descritiva das variáveis incluídas para interpretação dos grupos. A partir do teste ANOVA exibido na Tabela 4, foi possível concluir que as variáveis são significativamente diferentes, corroborando a existência de diferentes agrupamentos na indústria brasileira de confecções.

A partir da análise da Tabela 6 foi possível nomear os três grupos resultantes da análise de cluster em: grupo 1 - "Grandes - alto desempenho"; grupo 2 - "Pequenas - baixo desempenho"; e grupo 3 - "Pequenas - desempenho moderado".

Para suportar as diferenças entre porte dos três agrupamentos, foi realizado um teste de Quiquadrado entre o porte e os grupos estratégicos, descrito na Tabela 7.

\subsubsection{Grupo 1 - Grandes - alto desempenho (GAD)}

Este grupo é formado por 5\% das organizações que compõem a amostra. De acordo com a Tabela 7, verifica-se que, das empresas deste grupo, predominam as de grande porte, principalmente, em razão da média obtida para pessoal ocupado, mostrada na Tabela 6. Dos resultados disponibilizados nas Tabelas 5 e 6, foi possível inferir que este grupo possui as empresas mais eficientes tecnicamente. Também, pode-se perceber que esse grupo possui maior giro e maior retorno (ROA).

Já era esperado destas empresas giro elevado, visto que a alta concorrência dificulta a elevação das margens de lucro, fazendo com que o giro seja variável importante para obtenção de retorno. Como afirmou Matarazzo (2008), uma empresa pode obter rentabilidade pela margem ou, então, no caso de baixa margem de lucro, ganhar rentabilidade pelo giro das vendas, não descartando a possibilidade de aumento de rentabilidade pelo aumento da margem e giro simultaneamente. Como citado por Agis, Gouveia e Vaz (2002), Corrêa e Pimenta (2006) e Aslesen (2008), o setor confeccionista de grande porte deve ganhar em economia de escala com a padronização

Tabela 4. ANOVA para as variáveis utilizadas na interpretação dos clusters.

\begin{tabular}{lccc}
\hline & ANOVA & F & Significância \\
\hline Pessoal ocupado & 3,988 & 0,019 \\
Rentabilidade do ativo (ROA) & 3,111 & 0,045 \\
Giro do ativo & 7,111 & 0,001 \\
Margem líquida & 5,847 & 0,003 \\
\hline
\end{tabular}

Fonte: resultados da pesquisa. 
Tabela 5. Análise descritiva de eficiência por cluster.

\begin{tabular}{clcccrc}
\hline Cluster & $\begin{array}{c}\text { Escores de } \\
\text { eficiência }\end{array}$ & $\begin{array}{c}\text { eficientes } \\
(\boldsymbol{\%})\end{array}$ & Média & Mínimo & Máximo & $\begin{array}{c}\text { Desvio } \\
\text { padrão }\end{array}$ \\
\hline \multirow{2}{*}{1} & RCE & 0,00 & 41,06 & 13,8 & 57,90 & 13,03 \\
& RVE & 60,00 & 87,09 & 34,00 & 100,00 & 18,94 \\
& ESCALA & 0,00 & 46,88 & 24,72 & 62,56 & 10,49 \\
\hline \multirow{2}{*}{2} & RCE & 0,00 & 35,41 & 8,10 & 49,00 & 8,22 \\
& RVE & 0,00 & 37,92 & 8,30 & 60,90 & 9,03 \\
& ESCALA & 82,60 & 93,84 & 54,55 & 100,00 & 7,21 \\
\hline \multirow{2}{*}{3} & RCE & 13,00 & 65,57 & 41,70 & 100,00 & 16,72 \\
& RVE & 22,30 & 71,63 & 48,30 & 100,00 & 17,40 \\
\hline
\end{tabular}

Fonte: resultados da pesquisa.

Tabela 6. Análise descritiva das variáveis inseridas na análise de cluster.

\begin{tabular}{|c|c|c|c|c|c|c|}
\hline Cluster & Variáveis & $\begin{array}{c}\text { Amostra } \\
(\%)\end{array}$ & Média & Mínimo & Máximo & $\begin{array}{l}\text { Desvio } \\
\text { padrão }\end{array}$ \\
\hline \multirow{4}{*}{1} & Pessoal ocupado & \multirow{4}{*}{5} & $1.095,60$ & 2,00 & $10.059,00$ & $2.129,79$ \\
\hline & Retorno sobre o ativo (ROA) & & 19,39 & $-134,47$ & 593,25 & 123,52 \\
\hline & Giro do ativo & & 112,57 & 0,15 & 985,71 & 211,18 \\
\hline & Margem líquida & & 0,01 & $-0,58$ & 0,59 & 0,26 \\
\hline \multirow{4}{*}{2} & Pessoal ocupado & \multirow{4}{*}{53} & 66,55 & 2,00 & 529,00 & 59,70 \\
\hline & Retorno sobre o ativo (ROA) & & $-5,71$ & $-1173,83$ & 35,53 & 73,73 \\
\hline & Giro do ativo & & 31,88 & 0,00 & 1325,81 & 122,14 \\
\hline & Margem líquida & & $-0,06$ & $-3,93$ & 0,71 & 0,47 \\
\hline \multirow{4}{*}{3} & Pessoal ocupado & \multirow{4}{*}{42} & 124,25 & 1,00 & 236,00 & 229,63 \\
\hline & Retorno sobre o ativo (ROA) & & 7,75 & $-73,53$ & 481,94 & 54,93 \\
\hline & Giro do ativo & & 40,63 & 0,00 & 5249,39 & 366,87 \\
\hline & Margem líquida & & 0,25 & $-34,66$ & 0,67 & 2,44 \\
\hline
\end{tabular}

Fonte: resultados da pesquisa.

Tabela 7. Quiquadrado entre porte e grupos estratégicos.

\begin{tabular}{clccc}
\hline & & \multicolumn{3}{c}{ Cluster } \\
\cline { 3 - 5 } & & $\mathbf{1}(\boldsymbol{\%})$ & $\mathbf{2}(\boldsymbol{\%})$ & $\mathbf{3}(\boldsymbol{\%})$ \\
\hline \multirow{3}{*}{ Porte } & MPEs & 20 & 84 & 68 \\
& Médio porte & 28 & 15 & 28 \\
& Grande porte & 52 & 1 & 4 \\
\hline
\end{tabular}

$\chi^{2}=112,257 ; \mathrm{p}$-valor $=0,000$. Fonte: resultado da pesquisa.

dos produtos e a redução do custo unitário. Dessa maneira, essas empresas concorrem para a redução do preço do produto, exigindo maior giro do que margem. O ganho em rentabilidade obtido pela margem, segundo os autores deste artigo, deve ser relevado aos esforços das MPEs que buscam pela diferenciação de seus produtos e mercado de nicho. Apesar de não possuírem capacidade de ganhos de escalas, possuem maior flexibilidade para diferenciação e para as mudanças de acordo com tendência da moda. Com isso, as MPEs podem agregar valor aos produtos e buscar ganhos de margem ao invés de ganhos em giro.
O fato deste grupo possuir maiores médias para os indicadores de rentabilidade, giro e eficiência técnica mostra que as grandes empresas têm demonstrado melhor desempenho do que as MPEs, baseando-se no Quiquadrado evidenciado na Tabela 7 e nas médias para pessoal ocupado da Tabela 6. Baseando-se nas conclusões de Shergill (2002), Nickerson (2008) e outros estudiosos do mesmo tema, esse grupo cria, com isso, barreiras de mobilidade que o protege da concorrência interna no mesmo setor ou da entrada de concorrentes externos. Sabe-se que estas empresas possuem maiores recursos para investir em propaganda, força de vendas do que as MPEs, sendo isso uma 
barreira natural criada pela diferença de porte e disponibilidade de recursos financeiros.

Killstrom (2005) mostrou nos seus estudos a diferença de rentabilidade, retorno e eficiência dentro de uma mesma indústria. Como se pode perceber, a mesma conclusão pode ser considerada para a indústria de confecções brasileira.

\subsubsection{Grupos 2 - Pequenas - baixo desempenho (PBD)}

Pela Tabela 7, percebe-se que o grupo é formado por $84 \%$ de MPEs, corroborando a hipótese levantada e discutida para o primeiro grupo de que o desempenho e eficiência do setor confeccionista brasileiro são diferentes entre os portes. A classificação destas organizações neste grupo corrobora a afirmação da Abravest (ASSOCIAÇÃO..., 2009) sobre a composição do setor confeccionista brasileiro, principalmente, por MPEs.

A menor eficiência desse agrupamento pode ser explicada em razão destas não estarem agregando valor suficiente em decorrência da escassez de tecnologia e a utilização de mão de obra pouco qualificada, citado por Agis, Gouveia e Vaz (2002) e Doig (2005). Outro ponto a ser destacado é que, ao contrário do Grupo 1, este grupo apresentou a maior média para eficiência de escala e mais de $80 \%$ das empresas são eficientes em escala, demonstrando grande utilização da capacidade disponível para produção.

A citação do Sebrae (2009) de que a indústria confeccionista brasileira volta-se praticamente para o mercado interno pode ser uma das causas para a baixa competitividade brasileira frente aos produtos estrangeiros. Pode-se considerar que as cotas de importação fizeram com que o setor se acomodasse em um status quo ineficiente e não buscasse manter os mesmos padrões de competitividade internacional, atendendo somente à demanda interna para esses produtos.

A composição da indústria confeccionista por MPEs com mão de obra pouco qualificada e pouca tecnologia mostra que o Brasil segue o caminho informalmente denominado por Woll et al. (2007) como "estrada baixa". Isso conduz à competitividade ineficiente, a qual, segundo Brodner e Latniak (2002), leva a problemas de longo prazo provocados por baixos salários e baixo desempenho.

Como já discorrido para o primeiro grupo, as grandes empresas possuem mais condições para concorrência focada em custos pela possibilidade de ganhos em escala. As MPEs, no entanto, possuem melhores condições para buscar ganhos em margem pela diferenciação de produtos ou atendimento aos nichos de mercado como forma de melhorar o desempenho dentro do setor e, com isso, criarem barreiras de mobilidade intragrupo.
Apesar de Moniz e Paulos (2008) e Sebrae (2009) afirmarem avanços tecnológicos na fase anterior ao corte, sabe-se que, na maioria das MPEs, as confecções possuem pouca ou nenhuma tecnologia. Assim, a pouca qualificação da mão de obra, característica deste setor, conduz ao ineficiente aproveitamento dos recursos, elevação do custo unitário dos produtos e perda de competitividade para produtos importados. Isso evidencia que as considerações de Saraiva, Pimenta e Corrêa (2001), Woll et al. (2007), Moniz e Paulos (2008) e Unicamp (UNIVERSIDADE..., 2008) estão corretas sobre a invasão de confeccionados importados no mercado brasileiro em decorrência da baixa competitividade da indústria nacional.

Segundo Corrêa e Pimenta (2006), Aslesen (2008) e Moniz e Paulos (2008), existe um processo de deslocamento das fases de costura e montagem para países em desenvolvimento. Porém, no Brasil, percebe-se uma indústria que busca atender ao mercado interno, perdendo em competitividade, em razão dos procedimentos de subcontratação das grandes empresas confeccionistas internacionais.

A grande quantidade de MPEs nesse setor pode ser explicada pela fragilidade às barreiras de entrada, segundo Brodner e Latniak (2002) e Nordas (2004). Pode-se considerar este um grupo estratégico instável e desprotegido pelas barreiras de mobilidade.

Desta maneira, sugerem-se estratégias alternativas, para que esse grupo possa diferenciar-se dos outros grupos, nos quais predominam as grandes empresas, como discutido por Porter (1990) e Ingram e Yue (2008). Assim, as MPEs encontrarão caminho na especialização, diferenciação, enquanto as grandes empresas, na produção de larga escala e baixo custo, como afirmou Agis, Gouveia e Vaz (2002). Ainda, as MPEs podem buscar enfoques cooperativos como alternativa estratégica.

Analisando as discussões de Porter (1990) e Ingram e Yue (2008) percebe-se que este grupo deveria buscar estratégias de atuação que eliminasse a necessidade de economia de escala, como a atuação em mercados limitados aos pequenos nichos. Isso como forma de evitar a competição direta com os produtos padronizados estrangeiros. A diferenciação criaria barreira de mobilidade interna e externa, protegendo o grupo da vulnerabilidade, bem como auxiliando na redução dos índices de mortalidade de MPEs, em razão das exigências de investimentos iniciais impostos pelas barreiras de entradas, conforme as alternativas estratégicas aqui discutidas.

\subsubsection{Grupo 3 - Pequenas - desempenho moderado (PDM)}

Este grupo foi formado por $42 \%$ da amostra e, como se pode verificar na Tabela 7, possui $96 \%$ da amostra composta por MPEs e médias empresas, 
Tabela 8. Wilk's Lambda.

\begin{tabular}{lccc}
\hline & Wilks' Lambda & F & Significância \\
\hline RCE & 0,425616 & 232,7945 & 0,00 \\
RVE & 0,343836 & 329,1923 & 0,00 \\
ESCALA & 0,425109 & 233,2784 & 0,00 \\
\hline
\end{tabular}

Fonte: resultados da pesquisa.

Tabela 9. Coeficientes discriminantes.

\begin{tabular}{lcc}
\hline & Função 1 & Função 2 \\
\hline EFTRC & $-0,19$ & 0,20 \\
EFTRV & 0,22 & $-0,12$ \\
EFESCALA & 0,08 & $-0,07$ \\
Constante & $-9,94$ & 3,72 \\
\hline
\end{tabular}

Fonte: resultados da pesquisa.

Tabela 10. Probabilidades da função discriminante.

\begin{tabular}{|c|c|c|c|c|c|}
\hline & Cluster & $1(\%)$ & $2(\%)$ & $3(\%)$ & Total (\%) \\
\hline \multirow{4}{*}{ Amostra de desenvolvimento } & 1 & 89 & 0 & 11 & 100 \\
\hline & 2 & 0 & 100 & 0 & 100 \\
\hline & 3 & 5 & 21 & 74 & 100 \\
\hline & 1 & 83 & 0 & 17 & 100 \\
\hline \multirow{3}{*}{ Cross-validated } & 2 & 0 & 99 & 1 & 100 \\
\hline & 3 & 5 & 21 & 74 & 100 \\
\hline & 1 & 71 & 29 & 0 & 100 \\
\hline \multirow{2}{*}{ Amostra de validação } & 2 & 0 & 99 & 1 & 100 \\
\hline & 3 & 2 & 13 & 85 & 100 \\
\hline
\end{tabular}

Fonte: resultados da pesquisa.

com predominância para as MPEs. Esse grupo possui médias intermediárias para as variáveis pessoal ocupado, rentabilidade do ativo e giro do ativo e para a eficiência com RVE. Por outro lado, possui a maior média para margem líquida.

Este grupo não é totalmente predominante em grandes empresas como o grupo 1 e também não é totalmente predominante em MPEs como o grupo 2. $\mathrm{O}$ que se nota é uma heterogeneidade maior do que nos outros grupos que possuem médias intermediárias para as proxys de desempenho utilizadas, exceto a maior média para margem líquida.

Provavelmente, essa heterogeneidade de diferentes portes foi o que desencadeou as médias intermédias para as variáveis de análise destes grupos. Essa observação também é importante para corroborar que o porte está positivamente correlacionado ao desempenho das empresas analisadas.

Diversos estudos sobre agrupamentos estratégicos encontram grupos intermediários que não demonstram características tão bem definidas como os grupos inferiores ou superiores. Resultados como esses foram obtidos, por exemplo, nos estudos de Ferreira e Braga (2007b) e Ferreira, Abrantes e Perez (2008) na indústria de laticínios.

\subsection{Validação dos agrupamentos}

A análise discriminante foi utilizada para validação dos clusters como sugerido por Pestana e Gageiro (2000). Utilizaram-se os clusters formados como variável dependente e, como independentes, as variáveis utilizadas para interpretação dos grupos.

$\mathrm{O}$ procedimento stepwise manteve somente os escores de eficiência como variáveis discriminantes. No teste de igualdade de médias dos grupos, identificou-se que a eficiência por RVE é a melhor variável para discriminar os grupos, em função do baixo valor da estatística de Wilk’s Lambda (Tabela 8).

A confirmação final de que ocorreu ou não a quebra da premissa de igualdade entre as matrizes de covariância foi obtida por meio do teste Box's M que não foi violado por ter apresentado significância superior a 0,05. Desta forma, pode-se analisar adequadamente os coeficientes das funções exibidos na Tabela 9.

Ao analisar as médias da Tabela 10, com as probabilidades da análise discriminante, percebe-se que os casos da amostra de desenvolvimento foram $88 \%$ classificados corretamente, sendo que os casos do grupo 2 atingiram $100 \%$ de acerto. A amostra de 
cross-validated é o teste de Lamchembruch, em que cada caso da amostra de desenvolvimento é retirado da amostra cross-validated e uma nova função é gerada e aplicada para verificar a capacidade de classificação. Este teste mostrou um grau de acerto de $93 \%$ das classificações, sendo considerado plausível para uma boa diferenciação entre os grupos obtidos no estudo.

No geral, a função conseguiu classificar $87 \%$ dos casos não selecionados. Pode-se, então considerar que esse modelo tem capacidade para realizar, em um bom nível, a classificação de elementos externos a si, isto é, que não contribuíram para a sua composição. Dessa forma, esse modelo mostra que os clusters diferenciaram bem os grupos pelo desempenho.

Com isso, os resultados deste estudo demonstraram, significativamente, diferenciação em desempenho entre grupos estratégicos do mesmo modo que outros estudos mostraram essas diferenças (JOYCE, 2003; LEASK; PARKER, 2004 ; KILLSTROM, 2005).

\section{Conclusões}

Entre as principais contribuições para à literatura científica brasileira, este estudo propôs um modelo analítico de análise de desempenho do setor de confecções brasileiro, demonstrou a existência de grupos estratégicos que se diferenciam por seu desempenho e executou a validação do modelo proposto; muitas vezes, uma fase que não é destacada nos estudos que utilizam amostragem de dados em ciências sociais.

A presença de agrupamentos estratégicos diferenciados, principalmente por porte, mostra que o setor confeccionista nacional é dividido por barreiras internas inerentes às peculiaridades da maior disponibilidade de recursos em grandes empresas do que nas MPEs.

Portanto, os resultados corroboram a literatura especializada da área que aconselha as MPEs a focarem em outras estratégias que evitem a competição direta com as grandes empresas, principalmente em decorrência do menor aproveitamento de escala, devido às restrições de porte. Os resultados indicam ser mais apropriada às MPEs a construção de vantagens competitivas associadas à identificação de nichos de mercado não acessíveis aos produtos padronizados. Neste ponto, destaca-se que foi observado que, em média, nenhum dos grupos analisados se destacou pelo ganho em margem líquida.

Deixa-se então, neste estudo, a reflexão de que existe espaço para que confecções brasileiras busquem ganhos em margem e, com isso, possam, indiretamente, reduzir a concorrência com produtos padronizados internacionais de baixo custo. No entanto, existe a necessidade de se pensar em quais estratégias macros devem ser utilizadas para suprir as necessidades de mão de obra qualificada das MPEs e carência de tecnologia, para que estas possam atuar em mercados diferenciados.

É neste sentido que o grupo de menor desempenho, com predominância de micro e pequenas empresas, poderia melhorar consideravelmente seu desempenho relativo, evitando estratégias que conduzam à via de "estrada baixa". O caminho atualmente praticado fragiliza as barreiras de mobilidade deste grupo, cuja importância é indiscutível para a economia nacional. O fortalecimento das barreiras de mobilidade de cada agrupamento pode ser conduzido pelas estratégias discutidas na literatura deste estudo, bem como, com o posicionamento em economia de escala para as grandes empresas e pelo foco em pequenos nichos de mercado para as MPEs.

O baixo desempenho encontrado para o agrupamento composto basicamente por MPEs exige soluções urgentes e corretivas, principalmente, pela importância destas no cenário econômico nacional, tanto como empregador de mão de obra, quanto como mercado para produtos que não podem ser fabricados em larga escala. No entanto, este estudo reconhece que alguns fatores para qualificação da mão de obra, assim, como tecnologia, muitas vezes estão além das decisões dentro das empresas.

Os resultados indicam a possibilidade de intervenções qualitativas diferenciadas por agrupamento, por parte de organismos públicos ou privados no intuito de ampliar os níveis de desempenho do setor com consequências positivas para os elos subsequentes da cadeia. Além disso, medidas de adequação estratégica.

\section{Agradecimentos}

Os autores agradecem ao Instituto Brasileiro de Geografia e Estatística-IBGE por permitir a utilização dos dados da Pesquisa Industrial Anual de 2006.

\section{Referências}

ADHIKARI, R.; YAMAMOTO; Y. Textile and clothing industry: adjusting to the post-quota world. In: UNITED NATIONS. Department of Economic and Social Affairs. Industrial Development for the 21st Century: sustainable development perspectives. New York, 2007. p. 3-47.

AGIS, D.; GOUVEIA, J.; VAZ, P. Vestindo o futuro: macrotendências para as indústrias têxteis, vestuário e moda até 2020. Portugal: APIM, 2002.

ASLESEN, H. W. Prospective innovation challenges in the textiles and clothing sector. Europe INNOVA, 2008. Disponível em: <www.europe-innova.org/servlet/ Doc? cid=9953\&lg=EN>. Acesso em: 10 fev. 2009.

ASSOCIAÇÃO BRASILEIRA DA INDÚSTRIA TÊXTIL E DE CONFECÇÃO - ABIT. Seção Economia. Disponível em: <http://www.abit.org.br/site/navegacao.asp?id_ menu=8\&IDIOMA=PT $>$. Acesso em: 01 fev. 2009. 
A S S O C I A Ç ÃO B R A S ILEIR A D O VESTUÁRIO - ABRAVEST. Dados do setor. ABRAVEST, 2009. Disponível em: <http://www. abravest.org.br/>. Acesso em: 16 fev. 2009.

BANKER, R. D. Estimating most productive scale size using data envelopment analysis. European Journal of Operational Research, v. 17, p. 35-44, 1984. http:// dx.doi.org/10.1016/0377-2217(84)90006-7

BANKER, R. D.; CHARNES, A.; COOPER, W. W. Some models for estimating technical and scale inefficiencies in data envelopment analysis. Management Science, v. 30, n. 9, p. 1078-1092, 1984. http://dx.doi.org/10.1287/ mnsc.30.9.1078

BANKER, R. D.; THRALL, R. M. Estimation of returns to scale using DEA. European Journal of Operational Research, v. 62, n. 1, p. 74-84, 1992. http://dx.doi. org/10.1016/0377-2217(92)90178-C

BARNEY, J. B. Is the Resource-based "View" a Useful Perspective for Strategic Management Research? Yes. Academy of Management Review, v. 26, p. 41-56, 2001. BRODNER, P.; LATNIAK, E. The long way to the "High Road". Jahresbericht: IAT, 2002.

BURDEN, R.; PROCTOR, T. Creating a Sustainable Competitive Advantage through Training. Team Performance Management, v. 6, n. 5, p. 90-97, 2000. http://dx.doi.org/10.1108/13527590010348200

CHARNES, A.; COOPER, W. W.; RHODES, E. Measuring the efficiency of decision-making units. European Journal of Operational Research, v. 2, p. 429-444, 1978. http://dx.doi.org/10.1016/0377-2217(78)90138-8

CORRÊA, M. K.; PIMENTA, M. C. A. Reestruturação Produtiva na indústria do vestuário no município de Brusque - SC. Revista Discente Expressões Geográficas, v. 4, n. 2, p.84-98, 2006.

COUSINS, P. D. The Alignment of Appropriate Firm and Supply Strategies for Competitive Advantage. International Journal of Operations \& Production Management, v. 25, n. 5, p. 403-428, 2005. http:// dx.doi.org/10.1108/01443570510593120

DENRELL, J. The Performance of Performance. Journal of Management and Governance, v. 8, n. 4, p. 345-349, 2004. http://dx.doi.org/10.1007/ s10997-004-4127-x

DOIG, B. C. El mercado en la industria de la confección 15 años después: investigación cualitativa realizada con destacados empresarios de la industria de la confección de la ciudad de Medellín. Departamento de mercadeo, Escuela de Administración, mar. 2005. Disponivel em: $<$ http://www.eafit.edu.co/NR/rdonlyres/61263DA35A2F-4391-B9A5-8F37C75B8586/0/Cuaderno27.pdf>.

FAHY, J. The resource-based view of the firm: some stumbling-blocks on the road to understanding sustainable competitive advantage. Journal of European Industrial Training, v. 24, n. 2, p. 94-104, 2000. http://dx.doi. org/10.1108/03090590010321061

FAHY, J.; FARRELLY, F.; QUESTER, P. Competitive Advantage through Sponsorship: A Conceptual Model and Research Propositions. European Journal of Marketing, v. 38, n. 8, p. 1013-1030, 2004. http:// dx.doi.org/10.1108/03090560410539140
FERGUSON, T. D.; DEEPHOUSE, D. L.; FERGUSON, W. L. Do strategic groups differ in reputation? Strategic Management Journal, n. 21, p. 1195-1214, 2000. http://dx.doi.org/10.1002/10970266(200012)21:12<1195::AID-SMJ138>3.0.CO;2-R

FERREIRA, M. A. M.; BRAGA, M. J. Eficiência das sociedades cooperativas e de capital na indústria de laticínios. Revista Brasileira de Economia, v. 61, p. 231-244, 2007a.

FERREIRA, M. A. M.; BRAGA, M. J. Desempenho das cooperativas na indústria de laticínios do Brasil: uma abordagem por grupos estratégicos. RAUSP-Revista de Administração da Universidade de São Paulo, v. 42, p. 302-312, 2007b.

FERREIRA, M. A. M.; ABRANTES, L. A.; PEREZ, $R$. Investigação de grupos estratégicos na indústria de laticínios através da abordagem multivariada. RAM- Revista de Administração Mackenzie, v. 9, p. 152-172, 2008.

HAIR, J. F. Análise multivariada de dados. Porto Alegre: Bookman, 2005. 593 p.

INSTITUTO BRASILEIRO DE GEOGRAFIA E ESTATISTICA - IBGE. Séries estatísticas. Disponível em: <www.ibge.com.br>. Acesso em: 12 mai. 2009.

INGRAM, P.; YUE, L. Q. Structure, affect and identity as bases of organizational competition and cooperation. The Academy of Management Annals, v. 2, n. 1, p. 275-303, 2008. http://dx.doi. org/10.1080/19416520802211578

JOHNSON, A. J. et al. Strategic Group Analysis of U.S. Food Businesses Using the Two-step Clustering Method. International Food and Agribusiness Management Review, v. 14, n. 2, p. 83-102, 2011.

JOYCE, W. F. Integrating the resource-based and strategic groups influences on firm performance: extending a meso perspective. Research in Multi-Level Issues, v. 2, p.189-197, 2003. http://dx.doi.org/10.1016/ S1475-9144(03)02011-3

KILLSTROM, P. Strategic groups and performance of the firm: towards a new competitive environment in the Finnish telecommunications industry. 2005. Dissertation (Master)-Helsinki School of Economics, Espoo, 2005.

LEASK, G.; PARKER, D. Strategic groups, competitive groups and performance within the UK pharmaceutical industry: improving our understanding of the competitive process. Aston Business School, Aston University, 2004. The research director.

LEE, J.; LEE, K.; SANGKYU, R. An Evolutionary Perspective on Strategic Group Emergence: A Genetic Algorithm-Based Model. Strategic Management Journal, v. 23, p. 727-746, 2002. http://dx.doi. org/10.1002/smj. 250

LEONE, N. M. C. P. G. As Especificidades das pequenas e médias empresas. Revista de Administração, São Paulo, v. 34, n. 2, p. 91-94, 1999.

LIAO, S. H.; HU, T. C. Knowledge Transfer and Competitive Advantage on Environmental Uncertainty: An Empirical Study of the Taiwan Semiconductor Industry. Technovation, v. 27, p. 402-411, 2007. http://dx.doi. org/10.1016/j.technovation.2007.02.005 
LIN, B. W. Technology Transfer as Technological Learning: A Source of Competitive Advantage for Firms With Limited R\&D Resources. R\&D Management, v. 33, n. 3, p. 327-341, 2003. http:// dx.doi.org/10.1111/1467-9310.00301

MA, H. Competitive Advantage and Firm Performance. Competitiveness Review, v. 10, n. 2, p. 15-32, 2000.

MATITZ, Q. R. S.; BULGACOV, S. O conceito desempenho em estudos organizacionais e estratégia: um modelo de análise multidimensional. Revista de Administração Contemporânea, v. 15, n. 4, 2011.

MAROCO, J. Análise estatística. Lisboa: Sílabo, 2003. 508 p.

MATARAZZO, D. C. Análise financeira de balanços: abordagem básica e gerencial. 6. ed. São Paulo: Atlas, 2008.

McNAMARA, G.; DEEPHOUSE, D. L.; LUCED, R. A. Competitive positioning within and across a strategic group structure: the performance of core, secondary, and solitary firms. Strategic Management Journal, v. 24, p. 161-181, 2003. http://dx.doi.org/10.1002/smj.289

McGEE, J. Strategic Groups: theory and practice. In: FAULKNER, D. O.; CAMPBELL, A. (Eds.). The Oxford Handbook of strategy: a strategy overview and competitive strategy. 2003. v. 1, p. 261-301.

MONIZ, A.; PAULOS, M. R. The globalization in the clothing sector and its implications for work organization: a view from the Portuguese case. In: ISA FORUM OF SOCIOLOGY, 1., 2008, Barcelona. Proceedings... Barcelona, 2008. Disponível em: <http://mpra.ub.unimuenchen.de/10165/1/MPRA_paper_10165.pdf $>$. Acesso em: 12 fev. 2009.

NORDAS, H. K. The global textile and clothing industry post the agreement on textile and clothing. Geneva: World Trade Organization, 2004.

PELISSARI, A. S.; GONZALEZ, I. V. D. P.; VANALLE, R. M. Competências Gerenciais: um estudo em pequenas empresas de confecções. Revista Eletrônica de Administração-REAd, ed. 68, v. 67, n. 1, p. 149-180, 2011.

PESTANA, M. H.; GAGEIRO, J. N. Análise de dados para ciências sociais: a complementaridade do SPSS. 2. ed. Lisboa: Sílabo, 2000. 570 p.

PLESHKO, L. P.; NICKERSON, I. A. simple measure for the miles and snow strategy groups. Strategic Management Journal, v. 43, p. 135-148, 2008.
PORTER, M. E.; KRAMER, M. R. Strategy and Society: The Link between Competitive Advantage and Corporate Social Responsibility. Harvard Business Review, v. 84, n. 12, p. 78-92, 2006.

PORTER, M. The Competitive Advantage of Nations. New York: The Free Press, 1990.

RAMCHARRAN, H. Estimating productivity and returns to scale in the US textile industry. Empirical Economics, v. 26, p. 515-524, 2001. http://dx.doi.org/10.1007/ s001810000068

SARAIVA, L. A. S.; PIMENTA, S. M.; CORRÊA, M. L. faces do discurso empresarial no setor têxtil mineiro. Caderno de Pesquisa em Administração, v. 8, n. 2, p. 41-53, 2001.

SEBRAE. Têxtil e Confecções. 2009. Disponível em: <http://www.sebrae.com.br/setor/textil-e-confeccoes $>$. Acesso em: 16 fev. 2009.

SILVA, J. P. Análise financeira das empresas. São Paulo: Atlas, 2006.

SOUZA, R. M. Avaliação de custo, volume e lucro em micro e pequenas empresas comerciais: um estudo de caso. 2007. 107 f. Dissertação (Mestre em Engenharia de Produção)-Universidade Federal de Itajubá, Itajubá, 2007.

SHORT, J. C. et al. Firm, strategic group, and industry influences on performance. Strategic management journal, v. 28, p. 147-167, 2007. http://dx.doi. org/10.1002/smj.574

TAVARES, B.; FERREIRA, M. A. M.; LIMA, A. A. T. F. C. Identificação e agrupamento de fatores de relevância na investigação das práticas de gestão das micro e pequenas empresas. In: ENCONTRO DE ESTUDOS SOBRE EMPREENDEDORISMO E GESTÃO DE MICRO E PEQUENAS EMPRESAS, 5., 2008, São Paulo. Anais... São Paulo, 2008.

UNIVERSIDADE ESTADUAL DE CAMPINAS - UNICAMP. Instituto de Economia. Núcleo de Economia Industrial e da Tecnologia. Relatório de acompanhamento setorial: Têxtil e confecção. Campinas, 2008. Boletim de conjuntura industrial, acompanhamento setorial, panorama da indústria e análise da política industrial.

WOLL, T.; SILVA, A. V.; MONIZ, A. Organizational case study on design in the clothing and textile industry. Portugal: WORKS-Project, 2007. Internal Working Paper. 